\title{
Fusion Navigation Technique for Management of Difficult to Access Hepatocellular Carcinoma: A Single Center Experience
}

\author{
Ahmed M. El Nakib1", Sahar Zakria', Mohamed Hassany², Mahmoud El-Bendary ${ }^{1}$ \\ ${ }^{1}$ Tropical Medicine and Hepatogastroentrology Department, Mansoura Faculty of Medicine, Mansoura University, Mansoura, Egypt \\ ${ }^{2}$ National Hepatology and Tropical Medicine Research Institute, Cairo, Egypt \\ Email: ^el_naqueeb@yahoo.com
}

How to cite this paper: El Nakib, A.M., Zakria, S., Hassany, M. and El-Bendary, M. (2020) Fusion Navigation Technique for Management of Difficult to Access Hepatocellular Carcinoma: A Single Center Experience. Open Journal of Gastroenterology, 10, 329-340.

https://doi.org/10.4236/ojgas.2020.1012032

Received: November 24, 2020

Accepted: December 21, 2020

Published: December 24, 2020

Copyright $\odot 2020$ by author(s) and Scientific Research Publishing Inc. This work is licensed under the Creative Commons Attribution International License (CC BY 4.0).

http://creativecommons.org/licenses/by/4.0/

\section{(c) (i) Open Access}

\begin{abstract}
Introduction: Sonography is the most universally used imaging technique for planning and performing thermal ablation in Hepatocellular carcinoma patients due to its efficiency and safety. However, the presence of HCC nodules that are hardly visible on traditional sonography is a major drawback to its use during thermal ablation. Real-time image fusion (fusion imaging) or realtime virtual sonography is a new technology that has been developed. Aim: To determine the value of fusion/navigation guided percutaneous thermal ablation in the management of hepatocellular carcinoma that has poor conspicuity at conventional sonography. Subjects and Methods: This study included 70 HCC patients (BCLC A and B). Percutaneous radiofrequency ablation was done via real-time image fusion for 14 patients with poorly visible HCC nodules (study group), while Percutaneous radiofrequency ablation was done via traditional sonography for 56 patients with HCC nodules (control group). Results: The median time to reach the tumor was significantly shorter by using fusion navigation technique $(\boldsymbol{P}=\mathbf{0 . 0 3 4})$. By using fusion navigation technique $92 \%$ of the lesions were completely ablated while $55 \%$ only were completely ablated by using ultrasonography $(P=0.014)$. One year after the procedure, by using fusion navigation technique $92 \%$ of the patients had complete response and only $55 \%$ of the patients had complete response by using conventional ultrasonography $(P=\mathbf{0 . 0 1 1})$. The survival distributions for both interventions were statistically significantly different, $\chi^{2}=10.12, P=$ 0.001. Conclusion: Fusion imaging-guided percutaneous RFA is a reasonable and efficient treatment of patients with HCC undetectable by traditional ultrasonography.
\end{abstract}




\section{Keywords}

Fusion Navigation, Hepatocellular Carcinoma, Radiofrequency Ablation

\section{Introduction}

Hepatocellular carcinoma (HCC) is considered one of the poorest prognostic solid cancer, representing about $90 \%$ of primary liver cancer. It is the third most common cause of cancer related mortality worldwide. In spite of the recent development of the surgical techniques and new types of treatment, HCC is a disease with short-term survival [1].

In Egypt, HCC is considered a significant public health problem, where it is responsible for $33.63 \%$ and $13.54 \%$ of all cancers in males and females correspondingly [2] [3]. The process of carcinogenesis is complex involving multistep with different epigenetic, and genetic changes [4] [5].

The major risk factors for liver cancer in clinical practice are related to hepatitis C viral infection, hepatitis B viral infection, alcoholic and nonalcoholic fatty liver disease [6] [7] [8] [9]. The sequences of chronic HCV infection [10] [11] include the development of liver cirrhosis [12] [13], end stage liver diseases [14] and the progression of HCC depending on different immunological factors and genetic susceptibility [15] [16] [17] [18].

Alternative to surgical resection of HCC, Percutaneous thermal ablation is broadly performed as a non-invasive curative treatment for HCC [19].

For successful thermal ablation of HCC doctors must complete the following steps: planning, treatment (directing, monitoring, and controlling), and judging the treatment response [20].

Sonography is the most universally used imaging technique for planning and performing thermal ablation in HCC patients due to its efficiency and safety. However, the presence of hardly visible HCC nodules on traditional sonography is a major problem to its use during thermal ablation [21].

Real-time image fusion or real-time virtual sonography is a new technology has been progressed. It can reconstruct high-resolution multiplanar images by the use of 3D data, along with a position-tracking device which utilizes magnetic navigation. The real time sonograms could be joined with corresponding planar images taken by MRI, CT, or even sonographic volume data. Such new technology can overcome that weakness of traditional sonography [22].

By using this technique, we can reduce the sampling error by an accurate localization of the target lesions. Fusion imaging also improves the conspicuity of hepatocellular carcinomas (HCCs) and the feasibility of percutaneous radiofrequency ablation (RFA) of HCCs not identifiable on B-mode US [22].

Fusion imaging is also useful in avoiding the mistargeting of HCC-ablating a pseudolesion, not the true lesion-by reducing the false-positive detection of small 
HCCs on B-mode US. Fusion imaging can also guide accurate overlapping ablations of HCCs on the basis of the fused CT or MR images. Therefore, it can reduce the number of RFA sessions for HCCs invisible on B-mode US. Given that the detectability of HCCs depends on the tumor size, fusion imaging is likely to be more useful for small HCCs [22].

\section{Aim of Work}

The purpose of such study is to determine the value of fusion/navigation guided percutaneous thermal ablation in the treatment of HCC that has poor conspicuity at conventional sonography.

\section{Subjects and Methods}

\subsection{Patients}

Out of 1856 patients presented with hepatocellular carcinoma from January 2017 to May 2019, 70 patients from Tropical medicine and hepatogastroenterology department, Mansoura University Hospitals, Mansoura, Egypt and National Hepatology and Tropical medicine Research Institute, Cairo, Egypt were selected to be included in this study. After obtaining an approval from the ethical committee, a written consent was obtained from all patients, and the selected $70 \mathrm{pa}-$ tients with HCC were subdivided into two groups: The first group contained 14 patients who underwent radiofrequency thermal ablation with fusion navigation technique due to hard visibility of focal lesions by traditional ultrasonography and the second group contained 56 patients underwent radiofrequency thermal ablation via conventional ultrasonography. HCC diagnosis was done according to noninvasive diagnostic criteria obtained by imaging modalities [23]. Percutaneous radiofrequency ablation for both groups was done by two physicians with about 6 years of experience in thermal ablation as line of treatment of HCC.

Inclusion criteria for the study were patients with Child-Pugh class A or B, patients with prothrombin time ratio greater than $50 \%$ and platelet count greater than $50,000 / \mathrm{mm}^{3}$ and patients without portal vein thrombosis or extrahepatic metastasis.

All the patients were subjected to complete history taking, physical examination, laboratory investigations including complete blood picture, liver function tests (Serum albumin, Serum bilirubin Total\& Direct, SGPT, SGOT, Prothrombin Time.), virology (HBs Ag, HCV Ab), serum Creatinine and Alpha fetoprotein and radiological investigations (Pelvi-Abdominal ultasound and Dynamic Abdominal imaging studies (CT \&/MRI).

Out of 70 tumors included in this study, 14 tumors were considered not treatable with conventional US due to insufficient distinction of their echogenicity from adjacent tissues or inability to be detected, as they were sited in an inaccessible area by US as Contrast enhanced ultrasound (CEUS) is not available in our institution. 


\subsection{Technique}

\subsubsection{US-CT/MRI Imaging Fusion}

Ultrasound scanners (EIPIC, Philips) with convex probes of 3.5-MHz and needle guidance devices. Hardware involved a magnetic field transmitter sited near the right abdominal upper quadrant (PercuNav, Germany) and two electro-magnetic sensors, one being applied to the probe and the other being attached to the handle. This system allows orientation in space of the US probe.

CT/MRI data were then transmitted in DICOM format to the ultrasound system. Series for fusion imaging were chosen for each case depending on the optimal display criteria of targeted lesions and visualization of nearby blood vessels. US was registered initiating from a reference plane (often the trans-umbilical plane) as visualized by the two modalities. Fusion of images underwent further refining by the identification of $4-5$ concordant internal anatomical markers on both modalities with minimal adjusting to allow entire image alignment.

Recently, only one anatomical marker was required with "fine tuning" techniques. Acceptable registration could be determined in nearly five to 20 minutes. Then, the system undergoes simultaneous display in real-time ultrasound scans on a screen side with CT/MRI imaging on another side. A "virtual" target underwent manual application to the lesion visualized on CT-MRI scanning and a corresponding target was applied automatically to the corresponding site on US scan. The most convenient route to the targeted lesion was automatically applied on modalities.

\subsubsection{Ablation Procedures}

For both groups (70 patients) treatment was applied under general anesthesia. Radiofrequency ablation (RFA) was applied with a 200-Watt generator and single two-cm tip, 17-gauge monopolar, cooled-tip electrodes. For the $1^{\text {st }}$ group ( 14 patients), the ablation was done by fusion navigation technique while it was done via traditional ultrasonography for the $2^{\text {nd }}$ group (56 patients). For the $1^{\text {st }}$ group, applicator insertion was done at expiration or transient stopping of ventilation. During inserting the electrode, and all the time over the ablation, a virtual needle was shown on the CT-MRI scans.

\subsubsection{Postablation Targeting and Technical Success}

Follow up dynamic study (CT or MRI) was done one month after the procedure for both groups for detection of the outcome of ablation. All the studied groups were underwent Follow up over a period of one year for detection of any recurrence of HCC by dynamic study (CT or MRI).

\subsection{Data Analysis}

IBM's SPSS statistics (Statistical Package for the Social Sciences) for windows (version 25, 2017) was used for statistical analysis of the collected data. Shapiro-Wilk test was used to check the normality of the data distribution. All tests were conducted with $95 \%$ confidence interval. $P$ (probability) value $<0.05$ was 
considered statistically significant. Charts were generated using SPSS' chart builder and Microsoft Excel for windows 2019. A survival analysis was conducted using Kaplan-Meier graph to assess the median duration for in preventing recurrence in the treated patients. Reading from both groups were compared using the log rank test.

\section{Results}

As illustrated in Table 1, the $1^{\text {st }}$ group (study group) contained 14 patients (10 males and 4 females) while the $2^{\text {nd }}$ group (control group) contained 56 patients (48 males and 8 females) $(P=0.24)$. The median age of the study group was $59 \pm$ 5.6 years while it was $60.04 \pm 5.1$ years in the control group $(P=0.63)$. There was no significant difference between both groups as regard etiology of liver disease $(P=0.58)$. There was no significant difference between study and control group as regard presence of cirrhosis, Child pugh classification and BCLC classification ( $P=1,0.05$ and 0.34 respectively).

As illustrated in Table 2, the study group contained 12 patients with single lesion and only 2 patients had 2 lesions while 53 patients had single lesion and only 3 patients had 2 lesions in the control group $(P=0.58)$. The median of the maximum diameter of the tumor was $1.8 \pm 0.28 \mathrm{~cm}$ in the $1^{\text {st }}$ group and $1.84 \pm$ $0.26 \mathrm{~cm}$ in the $2^{\text {nd }}$ group $(P=0.61)$. There was no significant difference between the two groups as regard tumor location and history of previous ablation $(P=$ 0.66 and 1 respectively).

As regard tumor visibility and accessibility in the study group tumor, $64 \%$ were not visible by conventional ultrasound and $36 \%$ were hardly visible. As regard Access route, $79 \%$ were difficult accessed and $21 \%$ were easily accessed (Table 3).

Table 1. Demographic data of all patients.

\begin{tabular}{|c|c|c|c|c|c|}
\hline & & Stu & $u p(n=14)$ & Control group $(\mathrm{n}=56)$ & $P$ value \\
\hline \multicolumn{2}{|c|}{ Age } & \multicolumn{2}{|c|}{$59.29 \pm 5.65$} & $60.04 \pm 5.11$ & 0.63 \\
\hline \multirow{2}{*}{ Sex } & Male & & $(10)$ & $86 \%(48)$ & \multirow{2}{*}{0.24} \\
\hline & Female & & $\%(4)$ & $14 \%(8)$ & \\
\hline \multirow{2}{*}{$\begin{array}{l}\text { Etiology of } \\
\text { liver disease }\end{array}$} & $\mathrm{HBV}$ & & $\%(2)$ & $5 \%(3)$ & \multirow{2}{*}{0.58} \\
\hline & $\mathrm{HCV}$ & & $(12)$ & $95 \%(53)$ & \\
\hline \multicolumn{2}{|c|}{ Presence of cirrhosis } & \multicolumn{2}{|c|}{$100 \%(14)$} & $100 \%(56)$ & 1 \\
\hline \multirow{2}{*}{\multicolumn{2}{|c|}{ Child classification }} & A & $64 \%(9)$ & $88 \%(49)$ & \multirow{2}{*}{0.05} \\
\hline & & B & $36 \%(5)$ & $12 \%(7)$ & \\
\hline \multirow{2}{*}{\multicolumn{2}{|c|}{ BCLC classification }} & A & $79 \%(11)$ & $91 \%(51)$ & \multirow{2}{*}{0.34} \\
\hline & & B & $21 \%(3)$ & $9 \%(5)$ & \\
\hline
\end{tabular}

Data is expressed as percentage and frequency. 95\%CI: 95\% confidence interval of difference. $P$ is significant when $<0.05$ 
Table 2. Tumor characteristics and treatment history of both groups.

\begin{tabular}{lcccc}
\hline & & Study group $(\mathrm{n}=14)$ & Control group $(\mathrm{n}=56)$ & $P$ value \\
\hline \multirow{2}{*}{ Number of lesions } & 1 & $86 \%(12)$ & $95 \%(53)$ & 0.58 \\
\multirow{2}{*}{ Tumor maximum diameter } & $14.3 \%(2)$ & $5 \%(3)$ & 0.61 \\
& 2 & $7 \%(1)$ & $1.84 \pm 0.26$ & \\
IV & $50 \%(7)$ & $12 \%(6)$ & 0.06 \\
Location & V & $21 \%(3)$ & $50 \%(28)$ & \\
VI & $14 \%(2)$ & $18 \%(10)$ & 1 \\
\hline
\end{tabular}

Data is expressed as percentage and frequency. $95 \%$ CI: $95 \%$ confidence interval of difference. $P$ is significant when $<0.05$.

Table 3. Tumor characteristics of the study group.

\begin{tabular}{ccc}
\hline & & Study group $(\mathrm{n}=14)$ \\
\hline \multirow{2}{*}{ Tumor visibility } & Not visible & $64 \%(9)$ \\
& Hardly visible & $36 \%(5)$ \\
& Visible & $0 \%$ \\
\hline \multirow{2}{*}{ Access route } & Difficult & $79 \%(11)$ \\
& Easy & $21 \%(3)$ \\
\hline
\end{tabular}

As shown in Table 4, one patient was excluded from the first group asfusion navigation technique was inaccurately performed and the patient underwent laparoscopic RF. The median time to reach the tumor was $1.85 \pm 0.98$ minutes in the $1^{\text {st }}$ group and $2.57 \pm 1.26$ minutes in the $2^{\text {nd }}$ group $(P=0.034) .92 \%$ of the patients had complete ablation in the $1^{\text {st }}$ group while only $55 \%$ of the patients had complete ablation in the $2^{\text {nd }}$ group after one month of the procedure $(P=0.014)$. As regard the technical efficacy after one year of the procedure, $92 \%$ of the patients had complete response in the $1^{\text {st }}$ group while only $54 \%$ of the patients had complete response in the $2^{\text {nd }}$ group $(P=0.011)$. There was no need to perform artificial ascites in the $1^{\text {st }}$ group while 3 patients in the $2^{\text {nd }}$ group needed artificial ascites to complete the procedure $(P=0.62)$.

Kaplan-Meier survival analysis (Kaplan \& Meier, 1958) was conducted to compare the two different interventions for their effectiveness in preventing recurrence. Participants in the study group had a median time to recurrence of more than one year while those in the control group had a median survival time of 12 months. A log rank test was conducted to determine if there were differences in the survival distribution for the different types of intervention. The survival distributions for both interventions were statistically significantly different, $\chi^{2}=$ $14.02, p=<0.001$ (Figure 1). 
Table 4. Ablation technique of both groups.

\begin{tabular}{|c|c|c|c|c|}
\hline & & Study group $(\mathrm{n}=13)$ & Control group $(\mathrm{n}=56)$ & $P$ value \\
\hline \multicolumn{2}{|c|}{ Time to reach the tumor } & $1.85 \pm 0.987$ & $2.57 \pm 1.26$ & 0.034 \\
\hline \multicolumn{2}{|c|}{ Number of electrodes } & $1 \pm 0$ & $1 \pm 0$ & 1 \\
\hline & Not ablated & $0 \%$ & $9 \%(5)$ & \\
\hline \multirow[t]{3}{*}{ Ablation outcome } & Partial & $7 \%(1)$ & $36 \%(20)$ & 0.014 \\
\hline & Complete & $92 \%(12)$ & $55 \%(31)$ & \\
\hline & No response & $0 \%$ & $9 \%(5)$ & \\
\hline \multirow[t]{2}{*}{ Technical efficacy } & Partial & $7 \%(1)$ & $38 \%(21)$ & 0.011 \\
\hline & Complete & $92 \%(12)$ & $54 \%(30)$ & \\
\hline \multicolumn{2}{|c|}{ Artificial ascites } & $0 \%$ & $5 \%(3)$ & 0.62 \\
\hline
\end{tabular}

Data is expressed as percentage and frequency. $95 \%$ CI: $95 \%$ confidence interval of difference. $P$ is significant when $<0.05$.

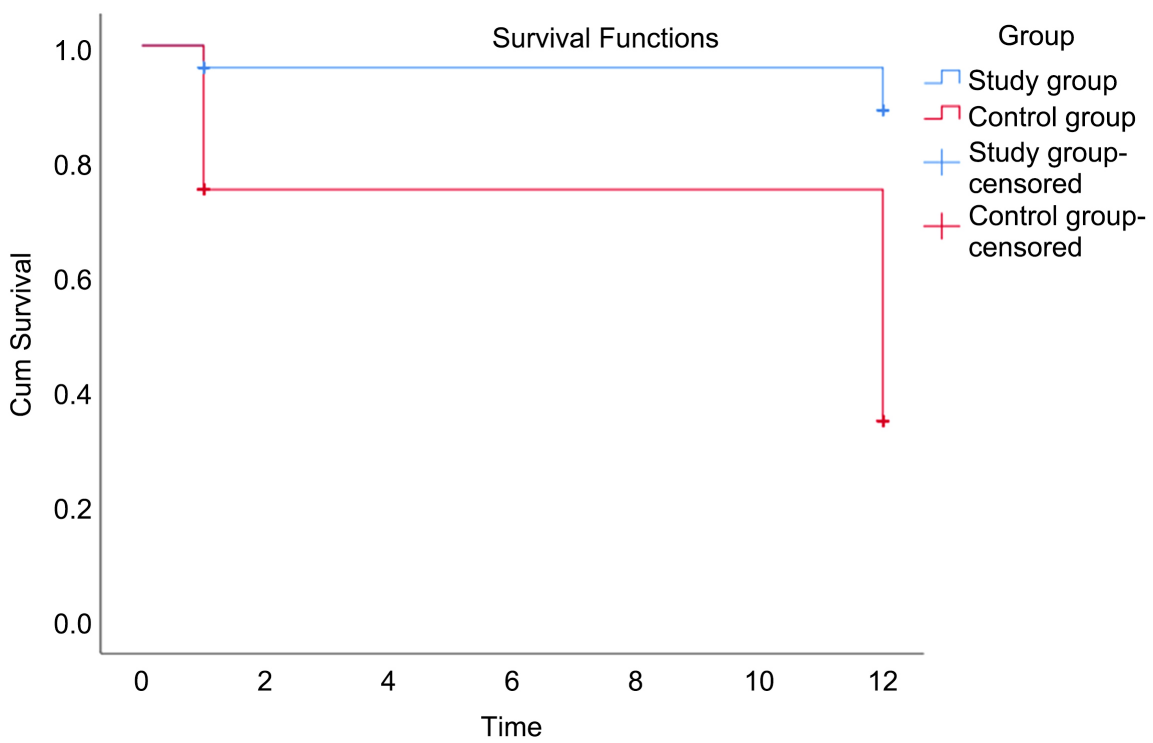

Figure 1. Kaplan-Meier survival analysis.

\section{Discussion}

The technical improvement of percutaneous thermal ablation might exert a large effect on the management of hepatocellular carcinoma (HCC), that is the sixth most prevalent cancer and the third main cause of cancer-related morality globally [24].

This type of treatment has been considered a main cornerstone in HCC management over the last 20 years, especially as advancement in contrast-enhanced CT (CECT) and contrast-enhanced MRI (CEMRI) has make the diagnosis of early stages of the disease easier [25].

Safe and efficient thermal ablation might require suitable imaging guidance throughout the process to permit visualizing the targeted lesions, surrounding structures, and needle probe itself [26]. 
Only recent reports of mixed populations of patients proving the accuracy and efficacy for real-time image fusion of US and CT have been published although the availability of this technique for some years [27].

In this study synchronization of B mode US images with the CT/MRI images using internal markers and RFA was done successfully under the guidance of the real-time navigation system for HCC cases.

Several documents dealing with thermal ablation of liver tumors guided by real-time virtual navigation systems have been published, but few of them deal with ablation of tumors invisible by ultrasound [27] [28] [29].

Real-time virtual navigation guided RFA was done for 14 HCCs (mean diameter was $1.8 \pm 0.28 \mathrm{~cm}$ ) with technical success rate of $92 \%$ evaluated with intravenous contrast-enhanced CT or MRI 1 month after ablation in this study, while $\mathrm{Liu}, \mathrm{Yu}$ [28] performed microwave thermal ablation of liver tumors guided by real-time virtual navigation technique for 18 HCCs $(0.9-3.8 \mathrm{~cm}$ in diameter) with technical success rate of $94.4 \%$.

Nakai, Sato [29] accomplished RFA for 20 HCCs (1.8 - 3.2 cm in diameter) in the CT set with a technical success rate of $90 \%$ (17/20 tumors).

In comparison with Mauri, Cova [26] who performed both RFA and MWA for 295 tumors undetectable with traditional US of smaller targets (mean diameter of $1.3 \pm 0.6 \mathrm{~cm}$ ) and the technical success rate was $90.2 \%$, we achieved a similarly high technical success rate (92\%).

This study revealed that the tumor visibility by B mode of ultrasound, out of 14 HCC nodules, 9 tumors (64\%) were not visible and 4 tumors (36\%) were hardly visible in contrast to previous study done by Mauri, Cova [26] who conducted that 295 of 1581 tumors (18.6\%) were undetectable with conventional ultrasonography.

Fusion navigation technique was inaccurately performed in one HCC patient (7\%) in this study and the patient has performed laparoscopic radiofrequency ablation and this was in contrast to the previous study performed by Mauri, Cova [26] who found that $13(4.4 \%)$ tumors were inaccurately targeting and it may be due to respiratory movements by the patients, needle bending, or incorrect co-registration of US and CT/MRI. These technical failure rate, despite being low, highlights that precise registration and organ motion might be crucial challenges to be dealt with by precise attention to technical details.

One HCC patient (7\%) was not completely ablated and this was similar to previous study done by who also revealed that one case was not ablated and this may be due to the effect of respiratory excursion and patient motion during insertion of the needle.

There was significant difference between patients treated by real-time image fusion of US and CT and patients treated by conventional ultrasound as regard technical success rate and this is similar to previous study done by Minami, Kudo [30] who found that the technical success rate after a single treatment session was significantly higher for the image fusion group (92\% versus $72 \%$ ). 
Similar to the results of this current study, Song, Lee [31] found that the technique effectiveness rate of radiofrequency ablation using fusion image was higher than those underwent treatment by conventional ultrasonography.

The technical efficacy of the procedure was assessed after 12 months of treatment and had $92 \%$ technical success rate, in contrast to $\mathrm{Xu}$, Long [32] who use both CT/MRI-CEUS and US-CEUS fusion imaging to evaluate treatment responses, all the lesions were treated with $100 \%$ technical success rates and complete ablation was achieved in 150 of 151 lesions (99.3\%) after 3 months of the procedure.

The recurrence of the tumor for the patient underwent treatment with conventional ultrasonography was much significantly higher than those patients underwent treatment by fusion imaging guidance and this may be due to adequate localization of the tumor by fusion imaging and thus adequate ablation success rate and efficacy and these results are similar to previous study done by Song, Lee [31].

Depending on adding the image-fusion that might lead to appreciation that the majority of cases successfully underwent even technically difficult thermal ablations in the US room using a modality characterized by simplicity, cheapness, availability and safeness.

Without this advance, a large number of those cases might have been underwent referral to highly invasive treatment methods (such as surgical resection) or to thermal ablation guided by complicated, high-cost, and less safe (because of the unsafety of irradiation) imaging modalities (CT and MRI). Thus, more cases with hepatic tumors that cannot be detected with US and CEUS might benefit from the accuracy of US-guided ablative treatments due to the real-time virtual navigation systems with fusion imaging.

This study had several limitations, first it is a single center study with small number of patients. Multiple center studies are needed to validate these results. Second the follow up period of the patients was only 1 year, thus, for hepatic malignancy we suggest future researches that can evaluate whether real differences in long-term local control and survival might be possible. Additionally, with more improvement, such image-guidance system might increase our abilities to apply ablation and other interventional modalities to more different patient populations.

\section{Conclusion}

Fusion navigation technique guided percutaneous RFA is effective for HCC with poor conspicuity at conventional ultrasound. Using this technique, it can increase operator confidence, the accuracy of the procedure, and technical success.

\section{Conflicts of Interest}

The authors declare no conflicts of interest regarding the publication of this paper. 


\section{References}

[1] Nikolova, D. (2019) Alpha Fetoprotein and Hepatocellular Carcinoma: An Opinion. Biomedical Journal of Scientific \& Technical Research, 12, 9555-9556. https://doi.org/10.26717/BJSTR.2019.12.002316

[2] Elghazaly, H., Gaballah, A. and Eldin, N.B. (2018) Clinic-Pathological Pattern of Hepatocellular Carcinoma (HCC) in Egypt. Annals of Oncology, 29, v5-v6. https://doi.org/10.1093/annonc/mdy151.018

[3] Elalfy, H., Besheer, T., El-Maksoud, M.A., Farid, K., Elegezy, M., El Nakib, A.M., Elsamanoudy, A.Z., et al. (2018) Monocyte/Granulocyte to Lymphocyte Ratio and the MELD Score as Predictors for Early Recurrence of Hepatocellular Carcinoma after Trans-Arterial Chemoembolization. British Journal of Biomedical Science, 75 187-191. https://doi.org/10.1080/09674845.2018.1494769

[4] Neamatallah, M.A., El-Missiry, M.A., Said, M.M., Elbendary, M., Othman, A.I. and Ammar, O.A. (2014) TP53 Polymorphism as a Risk Factor for Hepatocellular Carcinoma in Hepatitis C Virus-Infected Egyptian Patients. Egyptian Journal of Basic and Applied Sciences, 1, 9-15. https://doi.org/10.1016/j.ejbas.2014.01.001

[5] El-Bendary, M., Neamatallah, M., Abd El-Maksoud, M., El-Gendy, A. and El-Wehedy, A. (2015) Epidermal Growth Factor Genetic Polymorphism and Its Circulating Serum Level Predict the Risk of Hepatocellular Carcinoma in Egyptian Patients with HCV (Genotype-4)-Related Cirrhosis. International Journal of Advanced Research, 3, 697-705.

[6] Farid, K., Elalfy, H., Abo El-khair, S.M., Elgamal, H., Besheer, T., Elmokadem, A., El-Morsy, A., et al. (2020) Prognostic Value of Vascular Endothelial Growth Factor in Both Conventional and Drug Eluting Beads Transarterial Chemoembolization for Treatment of Unresectable Hepatocellular Carcinoma in HCV Patients. Expert Review of Gastroenterology \& Hepatology, 1-12. https://doi.org/10.1080/17474124.2020.1823215

[7] Besheer, T., Razek, A.A.K.A., El Bendary, M., Abd El-Maksoud, M., Elalfy, H., Zalata, K., El-Gilany, A.H., et al. (2017) Does Steatosis Affect the Performance of Diffusion-Weighted MRI Values for Fibrosis Evaluation in Patients with Chronic Hepatitis C Genotype 4. Turkish Journal of Gastroenterology, 28, 283-288. https://doi.org/10.5152/tjg.2017.16640

[8] El-Bendary, M., Hawas, S., El-Hammady, D., Al-Hadidy, A.H.M. and Eldegla, H. (2016) Profile of Expression of Certain Markers of Apoptosis in Chronic Hepatitis C and Hepatitis B Patients in an Egyptian Population. Archives of Virology, 161, 2369-2378. https://doi.org/10.1007/s00705-016-2897-5

[9] El-Bendary, M., Esmat, G., Neamatallah, M., Kamel, E., Besheer, T., Elalfy, H., Omran, D., et al. (2014) Epidemiological Aspects of Intrafamilial Spread of HCV Infection in Egyptian Population: A Pilot Study. Open Journal of Gastroenterology, 4, 228. https://doi.org/10.4236/ojgas.2014.45034

[10] Besheer, T., El-Bendary, M., Elalfy, H., Abd El-Maksoud, M., Salah, M., Zalata, K., Almashad, N., et al. (2017) Prediction of Fibrosis Progression Rate in Patients with Chronic Hepatitis C Genotype 4: Role of Cirrhosis Risk Score and Host Factors. Journal of Interferon \& Cytokine Research, 37, 97-102. https://doi.org/10.1089/jir.2016.0111

[11] Abdel-Azziz, M.Y., Zalata, K.R. and El-Bendary, M.M. (2010) Insulin Resistance and Liver Fibrosis Progression in Patients with Chronic Hepatitis C Virus Infection. Arab Journal of Gastroenterology, 11, 30-34.

https://doi.org/10.1016/j.ajg.2010.01.004 
[12] Besheer, T., Arafa, M., Abd El-Maksoud, M., Elalfy, H., Hasson, A., Zalata, K., Elsayed, E., et al. (2018) Diagnosis of Cirrhosis in Patients with Chronic Hepatitis C Genotype 4: Role of ABCB11 Genotype Polymorphism and Plasma Bile Acid Levels. The Turkish Journal of Gastroenterology, 29, 299. https://doi.org/10.5152/tjg.2018.17570

[13] Attallah, A.M., Omran, M.M., Farid, K., El-Bendary, M., Emran, T.M., Albannan, M.S. and El-Dosoky, I. (2012) Development of a Novel Score for Liver Fibrosis Staging and Comparison with Eight Simple Laboratory Scores in Large Numbers of HCV-Monoinfected Patients. ClinicaChimicaActa, 413, 1725-1730. https://doi.org/10.1016/j.cca.2012.06.031

[14] Abdel-Razik, A., Mousa, N., Abdel-Aziz, M., Elsherbiny, W., Zakaria, S., Shabana, W., El-Bendary, M., et al. (2019) Mansoura Simple Scoring System for Prediction of Spontaneous Bacterial Peritonitis: Lesson Learnt. European Journal of Gastroenterology \& Hepatology, 31, 1017-1024.

https://doi.org/10.1097/MEG.0000000000001364

[15] Neamatallah, M., El-Bendary, M., Elalfy, H., Besheer, T., El-Maksoud, M.A., Elhammady, D., Mousa, N., et al. (2020) Impact of Toll-Like Receptors 2 (TLR2) and TLR 4 Gene Variations on HCV Susceptibility, Response to Treatment and Development of Hepatocellular Carcinoma in Cirrhotic HCV Patients. Immunological Investigations, 49, 462-476. https://doi.org/10.1080/08820139.2019.1673772

[16] El-Bendary, M., Neamatallah, M., Elalfy, H., Besheer, T., Elkholi, A., El-Diasty, M. Elhammady, D., et al. (2018) The Association of Single Nucleotide Polymorphisms of Toll-Like Receptor 3, Toll-Like Receptor 7 and Toll-Like Receptor 8 Genes with the Susceptibility to HCV Infection. British Journal of Biomedical Science, 75, 175-181. https://doi.org/10.1080/09674845.2018.1492186

[17] El-Bendary, M., Neamatallah, M., Esmat, G., Kamel, E., Elalfy, H., Besheer, T., ElWaseef, A., et al. (2016) Associations of Human Leucocyte Antigen Class II-DQB 1 Alleles with Hepatitis C Virus Infection in Egyptian Population: A Multicentre Family-Based Study. Journal of Viral Hepatitis, 23, 961-970. https://doi.org/10.1111/jvh.12573

[18] El-Bendary, M., Neamatallah, M., Elalfy, H., Besheer, T., El-Setouhy, M., Youssef, M.M., Esmat, G., et al. (2019) Association of Genetic Polymorphisms of Chemokines and Their Receptors with Clearance or Persistence of Hepatitis C Virus Infection. British Journal of Biomedical Science, 76, 11-16. https://doi.org/10.1080/09674845.2018.1518299

[19] Tiong, L. and Maddern, G.J. (2011) Systematic Review and Meta-Analysis of Survival and Disease Recurrence after Radiofrequency Ablation for Hepatocellular Carcinoma. In: Database of Abstracts of Reviews of Effects (DARE): Quality-Assessed Reviews, Centre for Reviews and Dissemination (UK), York. https://doi.org/10.1002/bjs.7669

[20] Goldberg, S.N., Grassi, C.J., Cardella, J.F., Charboneau, J.W., Dodd, G.D., Dupuy, D.E., Livraghi, T., et al. (2005) Image-Guided Tumor Ablation: Standardization of Terminology and Reporting Criteria. Radiology, 235, 728-739.

[21] Kim, J.E., Kim, Y.S., Rhim, H., Lim, H.K., Lee, M.W., Choi, D., Cho, S.K., et al. (2011) Outcomes of Patients with Hepatocellular Carcinoma Referred for Percutaneous Radiofrequency Ablation at a Tertiary Center: Analysis Focused on the Feasibility with the Use of Ultrasonography Guidance. European Journal of Radiology, 79, e80e84. https://doi.org/10.1016/j.ejrad.2011.03.090

[22] Ewertsen, C., Săftoiu, A., Gruionu, L.G., Karstrup, S. and Nielsen, M.B. (2013) RealTime Image Fusion Involving Diagnostic Ultrasound. American Journal of Roent- 
genology, 200, W249-W255. https://doi.org/10.2214/AJR.12.8904

[23] Forner, A., Vilana, R., Ayuso, C., Bianchi, L., Solé, M., Ayuso, J.R., Brú, C., et al. (2008) Diagnosis of Hepatic Nodules $20 \mathrm{~mm}$ or Smaller in Cirrhosis: Prospective Validation of the Noninvasive Diagnostic Criteria for Hepatocellular Carcinoma. Hepatology, 47, 97-104. https://doi.org/10.1002/hep.21966

[24] Jemal, A., Bray, F., Center, M.M., Ferlay, J., Ward, E. and Forman, D. (2011) Global Cancer Statistics. CA: A Cancer Journal for Clinicians, 61, 69-90. https://doi.org/10.3322/caac.20107

[25] Park, H.J., Lee, J.M., Park, S.B., Lee, J.B., Jeong, Y.K. and Yoon, J.H. (2016) Comparison of Knowledge-Based Iterative Model Reconstruction and Hybrid Reconstruction Techniques for Liver CT Evaluation of Hypervascular Hepatocellular Carcinoma. Journal of Computer Assisted Tomography, 40, 863-871. https://doi.org/10.1097/RCT.0000000000000455

[26] Mauri, G., Cova, L., De Beni, S., Ierace, T., Tondolo, T., Cerri, A., Solbiati, L., et al. (2015) Real-Time US-CT/MRI Image Fusion for Guidance of Thermal Ablation of Liver Tumors Undetectable with US: Results in 295 Cases. Cardiovascular and Interventional Radiology, 38, 143-151. https://doi.org/10.1007/s00270-014-0897-y

[27] Di Mauro, E., Solbiati, M., De Beni, S., Forzoni, L., D’Onofrio, S. and Solbiati, L. (2013) Virtual Navigator Real-Time Ultrasound Fusion Imaging with Positron Emission Tomography for Liver Interventions. 2013 35th Annual International Conference of the IEEE Engineering in Medicine and Biology Society, Osaka, 3-7 July 2013, 1406-1409. https://doi.org/10.1109/EMBC.2013.6609773

[28] Liu, F.Y., Yu, X.L., Liang, P., Cheng, Z.G., Han, Z.Y., Dong, B.W. and Zhang, X.H. (2012) Microwave Ablation Assisted by a Real-Time Virtual Navigation System for Hepatocellular Carcinoma Undetectable by Conventional Ultrasonography. European Journal of Radiology, 81, 1455-1459. https://doi.org/10.1016/j.ejrad.2011.03.057

[29] Nakai, M., Sato, M., Sahara, S., Takasaka, I., Kawai, N., Minamiguchi, H., Takeuchi, N., et al. (2009) Radiofrequency Ablation Assisted by Real-Time Virtual Sonography and CT for Hepatocellular Carcinoma Undetectable by Conventional Sonography. Cardiovascular and Interventional Radiology, 32, 62-69. https://doi.org/10.1007/s00270-008-9462-x

[30] Minami, Y., Kudo, M., Kawasaki, T., Chung, H., Ogawa, C. and Shiozaki, H. (2004) Percutaneous Radiofrequency Ablation Guided by Contrast-Enhanced Harmonic Sonography with Artificial Pleural Effusion for Hepatocellular Carcinoma in the Hepatic Dome. American Journal of Roentgenology, 182, 1224-1226. https://doi.org/10.2214/ajr.182.5.1821224

[31] Song, K.D., Lee, M.W., Rhim, H., Cha, D.I., Chong, Y. and Lim, H.K. (2013) Fusion Imaging-Guided Radiofrequency Ablation for Hepatocellular Carcinomas Not Visible on Conventional Ultrasound. American Journal of Roentgenology, 201, 11411147. https://doi.org/10.2214/AJR.13.10532

[32] Xu, E., Long, Y., Li, K., Zeng, Q., Tan, L., Luo, L., Zheng, R., et al. (2018) Comparison of CT/MRI-CEUS and US-CEUS Fusion Imaging Techniques in the Assessment of the Thermal Ablation of Liver Tumors. International Journal of Hyperthermia, 35, 159-167. https://doi.org/10.1080/02656736.2018.1487591 